\title{
Optimum cultural conditions to achieve the best biofilm formation and high level icaA transcription by Staphylococcus aureus
}

\section{Aram Sharifi}

University of Kurdistan

Abdolmajid Mohammadzadeh ( $\square$ Mohammadzadeh@basu.ac.ir)

Bu-Ali Sina University

Pezhman Mahmoodi

Bu-Ali Sina University

Taghi Zahraei Salehi

Tehran University

\section{Research Article}

Keywords: Biofilm formation, Culture media, glucose, quantitative real-time RT-PCR, Staphylococcus aureus

Posted Date: October 12th, 2021

DOl: https://doi.org/10.21203/rs.3.rs-820845/v1

License: (c) (i) This work is licensed under a Creative Commons Attribution 4.0 International License. Read Full License 


\section{Abstract \\ Background}

The aim of this study was to investigate the influences of different broth culture media supplemented with glucose, on the biofilm formation and ica expression of Staphylococcus aureus. The phenotypic ability to adhere to a polystyrene surface and to produce slime layer were evaluated using microtiter plate test (MtP) and Congo red tube test, respectively. Using PCR, the presence of ica locus in S. aureus strains was confirmed and subsequently, quantitative real-time RT-PCR was performed to investigate transcription of $i c a A$ in various media including Tryptic soy broth (TSB), Brain-heart infusion broth (BHIB), (Nutrient broth) NB and (Muller-Hinton broth) MHB contained 0, 0.25, 0.5, 1 and 2\% glucose.

\section{Results}

Our results showed that although all of the studied strains adhered to the wells of polystyrene microtiter plates, the optimum rate of biofilm formation was observed for TSB medium contained $1 \%$ glucose, but biofilm formation was not significantly different in NB, MHB and BHIB media. Supplementation of all media with $1 \%$ glucose led to the highest production of biofilm formation and in all of media transcription of $i c a A$ was increased with glucose addition to one present.

\section{Conclusions}

The results of the present study indicated that TSB medium supplemented with $1 \%$ glucose was the most appropriate medium for evaluation of biofilm formation by $S$. aureus isolates.

\section{Background}

Biofilms is an adherent community of bacteria that irreversibly attached to a substratum or to each other. Biofilm infections, such as pneumonia in cystic fibrosis patients, chronic wounds, chronic otitis media and implant- and catheter-associated infections, affect millions of people in the developed world each year and many deaths occur as a consequence $[1,2]$. Structural analysis of biofilms often reveals that bacterial biofilms are often surrounded by an extracellular polymeric substance (EPS) [3]. The gene expression pattern of bacteria in a biofilm is different from planktonic cells. This difference in gene expression along with other characteristics including physical barrier of exopolysaccharides can cause increased tolerance against antibiotics, disinfectants, and host immune mechanisms in biofilms [4]. $S$. aureus is one of the most important bacterial species which can form biofilms on biotic and abiotic surfaces [5-7]. For instance, $S$. aureus biofilms may be involved in infections of central venous catheters, urinary catheters, prosthetic heart valves, orthopedic implants, and dialysis catheters. $S$. aureus EPS is mainly composed of poly- $\mathrm{N}$-acetylglucosamine polymer which is encoded by icaADBC operon $[7,8]$. 
Several phenotypic and genotypic methods are available for the detection of staphylococcal biofilms. Phenotypic methods such as MtP and Congo red agar (CRA) tests measure biofilm density and production of slime layer, respectively, while, genotypic methods like PCR, determine biofilm related genes e.g. those encoded microbial surface components recognizing adhesive matrix molecules (MSCRAMMs) $[9,10]$. MtP test uses 96 -well microtiter plates to measure the optical density (OD) values of stained bacterial biofilms. This method gives a quantitative result [9].

In CRA method, bacteria are cultured on Congo red agar and incubated; thereafter, the color of colonies indicates their ability to produce slime layer. Slime-forming strains appear as black colonies. whereas, non-slime-forming strains stain red [10]. In addition, the MSCRAMMs mediate $S$. aureus attachment to different surfaces [11]. Actually, intercellular signaling between bacteria, which is also known as quorum sensing, has been shown to be involved in the development of biofilm by $S$. aureus. Quorum-sensing occurs based on the synthesis and secretion of small hormone-like molecules (autoinducers). These molecules can bind to cognate receptors and contribute to biofilm formation. Quorum sensing in these bacteria is encoded by an accessory gene regulator (agr) locus. Activation of agr system can affect $S$. aureus biofilm formation through down-regulation of MSCRAMMs as well as production of a protease [12]. It has been described that bacterial species, attachment surface and surrounding medium are three important factors involved in the formation of biofilms [13]. The ability of each strain to adhere and form biofilm is very different in various culture media and environmental conditions [13], However, environmental factors such as $\mathrm{pH}$, temperature, osmolarity, $\mathrm{O}_{2}$ levels, nutrient composition and the presence of other bacteria can affect the ability of a microorganism to produce biofilm [14]. In this case, it has been shown in a study that glucose may repress agr system by lowering $\mathrm{pH}$ which results in increased biofilm formation [15]. Thus, the present study was conducted to introduce an appropriate bacterial culture medium with optimum glucose concentration for examination of the ability of biofilm formation by $S$. aureus strains.

\section{Results}

All of the isolates were genotypically confirmed as $S$. aureus. Figure 1 depicts the results of electrophoresis of the species-specific PCR.

\section{Microtiter Plate Assay (Dup: Abstract ?)}

The ability of biofilm formation was investigated by measuring OD values of the examined isolates using MtP assay. In all of the tested media, the biofilm formation rate of $S$. epidermidis 12228 was significantly lower than S. aureus 25923 and the other two isolates. However, the results revealed that no significant difference was observed in the rate of biofilm formation between S. aureus 25923 and the others. On the other hand, the results showed that the highest and lowest rates of biofilm formation occurred when bacteria were cultured in TSB and NB, respectively. The biofilm formation patterns for two clinical isolates were similar to what was seen for $S$. aureus 25923 (data are not shown). Addition of glucose from 0 to 
$1 \%$ in all media also showed a significant positive effect on the biofilm formation of $S$. aureus $(\mathrm{P}<0.05)$ and the most biofilm formation was observed in media with $1 \%$ glucose. However, increasing of glucose percentage up to 2 percent did not promote biofilm formation. Some reactions of biofilm formation in MtP test are shown in the Figure 2.

\section{Slime Assay (Dup: Abstract ?)}

In Congo red tube test, S. aureus 25923 and the other two clinical isolates (biofilm producer strains) changed the color of MHB, NB and TSB media from red to reddish grey after the incubation period. However, S. epidermidis 12228 did not change the base red color of all of the examined media. In other words, no significant difference was observed among these media in the test. Addition of glucose did not show any visible effect on the tested media (Figure 3).

\section{Real time-PCR for evaluation of icaA gene expression}

The results of the conventional PCR showed that ica $A$ was present in all three tested strains (two clinical isolates and $S$. aureus ATCC 25923). Sequencing of this PCR product confirmed the accuracy of PCR assay. The sequence obtained for $i c a A$ amplicon was $99 \%$ identical to the corresponding GenBank sequence (accession number WP_031785277.1). The results of real time-PCR indicated that expression of $i c a A$ was increased by addition of glucose to $1 \%$ in TSB, MHB, NB and BHIB media. Figure 4 depicts the results of real time PCR for $i c a A$ expression.

\section{Discussion}

The chemical components of growth media and their supplements such as $\mathrm{NaCl}$, ethanol, glucose and sub-inhibitory concentrations of antibiotics, strongly affect biofilm development [16]. In particular, the presence of glucose in the growth medium has been reported to enhance biofilm formation [17]. Various methods have been introduced to measure the biofilm-forming capability of $S$. aureus isolates, among which, MtP is one of the most common methods in bacteriology laboratory. In this technique, TSB medium containing various glucose concentrations is often used to study the adherence ability of $S$. aureus to 96-well polystyrene tissue culture plates [18].

In the present study, the ability of biofilm formation by $S$. aureus strains was investigated in four bacterial culture media (NB, TSB, MHB and BHIB) containing various glucose supplements $(0,0.25,0.5,1$ and $2 \%)$ using MtP assay. The results showed that among all of the tested broth media, TSB was the most suitable medium to examine the ability of biofilm formation. However, some studies reported that bacterial biofilm formation in BHIB is sometimes occurred better than TSB $[17,19,20]$. Nevertheless, it has also been shown that some staphylococcal strains produce greater biofilm in TSB compared to BHIB [21]. Moreover, researchers commonly use TSB with various glucose supplements for evaluation of biofilm formation by $S$. aureus strains [20]. It has been reported that addition of glucose to culture media lead to the increase of the ability of staphylococci biofilm formation $[19,21]$. In agreement with the 
previous findings, the present study showed significant increase in $S$. aureus biofilm production when the bacterium exposed to the increasing concentrations of glucose. The best concentration of glucose for biofilm formation of $S$. aureus on polystyrene surfaces in all of the tested culture media was $1 \%$.

In addition, Congo red tube test and real time-PCR were used to detect extracellular exopolysaccharide and demonstrate ica $A$ expression, respectively. RT-PCR results showed that ica $A$ was expressed in all of the media with different glucose concentrations but the most expression occurred when one percent glucose was added to the medium $(P<0.05)$. Within the icaADBC operon, the ica $A$ gene encodes the enzyme $\mathrm{N}$-acetylglucosaminyl transferase, capable to catalyse the synthesis of poly-N-acetylglucosamine polymer which is called PIA and is important for cell to cell adhesion and accumulation [22]. The icaADBC and products of the ica locus [icaR (regulatory) and icaADBC (biosynthetic) genes] have been demonstrated to be necessary for biofilm formation [23]. Consequently, in the present study, the expression of icaA locus was evaluated in different culture media supplemented with $0,0.25,0.5,1$ and $2 \%$ glucose. The results revealed that among all culture media (TSB, MHB, NB and BHIB) the most icaA expression level was detected in $1 \%$ glucose addition. In addition, the color of Congo red tube culture was not changed was not changed by glucose addition, indicating that this medium is not suitable for measurement of precise amount of PIA or measurement of icaADBC operon expression.

The results of the present study revealed that in fact, glucose increases the ability of biofilm formation by promoting icaA transcription and subsequently, PIA production. Hence, additional ica-independent factors may be involved in increasing of phenotypic biofilm formation by staphylococci. For instance, it was reported that Agr system is down-regulated by addition of glucose [15], and $S$. aureus agr mutants also show enhanced biofilm formation [24]. Therefore, glucose-induced promotion of biofilm formation may be related to a suppression mechanism of agr. Using phenotypic analysis of wild type and mutant strains of $S$. aureus, Lim et al also described that $r b f$ gene is another gene that mediates the biofilm formation of S. aureus at the multicellular aggregation stage in an ica independent manner [25], suggesting that the genetic basis of biofilm formation in staphylococci is multifactorial, and much remains to be explored.

\section{Conclusions}

The results of the present study suggested that among various examined media, TSB supplemented with $1 \%$ glucose was the most appropriate medium for evaluation of biofilm formation of $S$. aureus strains. However, further studies are necessary to elucidate the precise mechanisms of increasing $S$. aureus biofilm formation following glucose induction to culture media.

\section{Methods}

\section{Bacterial strains, culture media and growth conditions}

S. aureus ATCC 25923 was used as a reference biofilm producer strain in all of the experiments. Besides, two $S$. aureus strains isolated from tracheal tube samples of two patients ( 45 and 63 years old) admitted 
to the ICU of a hospital in Hamedan (west of Iran) were also included in the present study. These bacterial isolates were identified by culture characteristics and biochemical tests and their identity was also confirmed by a species-specific PCR assay which investigated femA gene of $S$. aureus [26]. Meanwhile, $S$. epidermidis ATCC 12228 was used as a non-biofilm producer control in the assays. All culture media were obtained from Merck (Darmstadt, Germany) and the bacteria were cultured under aerobic conditions at $37^{\circ} \mathrm{C}$ for $24 \mathrm{~h}$.

\section{Microtiter Plate Assay}

All $S$. aureus isolates were examined for the ability of biofilm formation using a previously described microtiter plate assay [18]. Briefly, $50 \mu \mathrm{l}$ of an overnight culture of each isolate was added into 96-well microplates (SPL, Korea) containing $50 \mu \mathrm{l}$ of each of the Nutrient broth (NB), Tryptic Soy broth (TSB), Mueller-Hinton broth (MHB), and Brain-heart infusion broth (BHIB) media supplemented with $0.25 \%, 0.5 \%$, $1 \%$ and $2 \%$ glucose (Merck, Germany). NB, TSB, MHB and BHIB media without glucose were also used as control group. The microplates were aerobically incubated at $37^{\circ} \mathrm{C}$ for 24 hours. The supernatants were then discarded and microplates were washed twice with phosphate-buffered saline (PBS). The plates were left to dry for an overnight and stained with $200 \mu \mathrm{l}$ of $0.1 \%$ Safranin dye for 15 min followed by three times washing with $300 \mu \mathrm{l}$ of PBS and air-dried for an overnight. Afterwards, $100 \mu \mathrm{l}$ of ethanol-acetic acid (95:5 V/V) was added to each well to dissolve bounded Safranin in adherent bacterial cells. Finally, optical density values were measured at $490 \mathrm{~nm}$ by a plate reader. The experiment was performed in triplicates. The biofilm-producer $S$. aureus ATCC 25923 and the biofilm non-producer $S$. epidermidis ATCC 12228 were used as control strains [27].

\section{Slime Assay}

Congo red tube test was performed to evaluate the ability of slime production in different media supplemented with various glucose concentrations. To do this, a single colony from an overnight culture was inoculated into $10 \mathrm{ml}$ of each of NB, TSB, MHB and BHIB media contained $0.04 \%$ Congo red dye and supplemented with $0.25,0.5,1$ and $2 \%$ glucose. NB, TSB, MHB and BHIB media without glucose were used as control group. The culture tubes were incubated on a shaker $(100 \mathrm{rpm})$ at $37^{\circ} \mathrm{C}$ for 24 hours. The cultured media were then categorized into three groups based on their colors: black, weak black and red. As exopolysaccharides directly react with Congo red and produce black color, the level of black color is considered as the amount of exopolysaccharides or polysaccharide intercellular adhesins (PIA) [28].Therefore, the black and red media were considered to be related to normal slime producing and nonslime producing isolates, respectively.

\section{Rna Extraction And Cdna Synthesis}


To evaluate transcription of $i c a A$ gene, the presence of its encoding DNA was first investigated in $S$. aureus isolates and the reference strain by a PCR assay using previously introduced primers [29]. The sequence of forward primer was 5'-TATTCAATTTACAGTCGCAC-3' and of reverse primer was 5'-

GATTCTCTCCCTCTCTGCCA-3'. These primers amplify a 407 bp DNA fragment of icaAD gene based on a published sequence (AF086783). A PCR product was sequenced to confirm PCR specificity. For removing DNA contamination, the extracted RNA was treated with RNase-free DNase I (Thermo Scientific, USA). The quality and quantity of the extracted RNA were determined by agarose gel electrophoresis and confirmed by measuring the absorbance at $260 \mathrm{~nm}$ using a Nanodrop spectrophotometer ND-1000 (Thermo Fisher Scientific, Wilmington, DE, USA). Extracted RNAs were stored at $-70^{\circ} \mathrm{C}$ for next experiments. Then, the purified RNA was converted to cDNA according to the manufacturer's instructions (cDNA Synthesis Kit, Takara, Japan), and stored at $-20^{\circ} \mathrm{C}$ to use as the template for real time RT-PCR.

\section{Relative Quantitative Real-time Rt-pcr}

SYBR Green real-time PCR Master Mix (Amplicon, Denmark) was used for real-time RT-PCR, according to the manufacturer's instructions. The primers (Takapouzist, Iran) used are listed in Table 1. The reactions were conducted in a Corbett Life Science Rotor-Gene 6000 Cycler (Qiagen, Germany) and 16S rRNA housekeeping gene was considered as an internal control to normalize the expression levels of $i c a A$. The amplification proceeded as follows: denaturation at $95^{\circ} \mathrm{C}$ for $10 \mathrm{~min}$ and then 40 cycles including denaturation at $95^{\circ} \mathrm{C}$ for $30 \mathrm{sec}$, annealing at $53^{\circ} \mathrm{C}$ (for icaA) and $59^{\circ} \mathrm{C}$ (for $16 S$ rRNA) for $30 \mathrm{sec}$, and $72^{\circ} \mathrm{C}$ for $30 \mathrm{sec}$. A negative control was included in each run. All the samples were analyzed in triplicate and finally, relative gene expression was calculated using the $2^{-\triangle \Delta C T}$ method [30].

Table I. Primers used for the quantitative real-time RT-PCR assay.

\begin{tabular}{|c|c|c|c|}
\hline Genes & 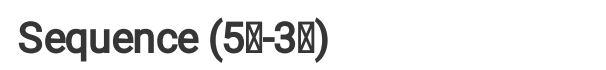 & Annealing temperature & Reference \\
\hline \multirow[t]{2}{*}{$i c a A$} & GGAAGTTCTGATAATACTGCTG & \multirow[t]{2}{*}{$53^{\circ} \mathrm{C}$} & \multirow[t]{2}{*}{ [31] } \\
\hline & GATGCTTGTTTGATTCCCTC & & \\
\hline \multirow[t]{2}{*}{$16 S \operatorname{rRNA}$} & AGCCGACCTGAGAGGGTGA & \multirow[t]{2}{*}{$59^{\circ} \mathrm{C}$} & \multirow[t]{2}{*}{ [32] } \\
\hline & TCTGGACCGTGTCTCAGTTCC & & \\
\hline
\end{tabular}

\section{Statistical Analysis}

One-way ANOVA was carried out to compare OD values obtained from three independent experiments using SPSS software. The mean difference was considered significant at $P<0.05$.

\section{Abbreviations}


MtP: microtiter plate test; TSB: Tryptic soy broth; BHIB: Brain-heart infusion broth; NB: Nutrient broth; MHB: Muller-Hinton broth; EPS: Extracellular polymeric substance; CRA: Congo red agar; MSCRAMMs: Microbial surface components recognizing adhesive matrix molecules; OD: Optical density; agr: Accessory gene regulator; PCR: Polymerase chain reaction

\section{Declarations}

\section{Ethics approval, guidelines and consent to participate}

This research was approved by the Ethics Committee for Research at Hamedan University of Medical Sciences with ethical approval No IR.UMSHA.REC.1395.145. In addition, informed consent form was obtained from all of the patients. All methods were performed in accordance with the relevant guidelines and regulations of national clinical testing operating procedures in Iran.

\section{Consent for publication}

Not Applicable.

\section{Availability of data and materials}

The raw data supporting the conclusions of this article will be made available by the corresponding author, without undue reservation.

\section{Competing interests}

The authors declare that they have no competing interests.

\section{Funding}

This research was supported by research grants (Grant NO: 95-200) from Bu-Ali Sina University of Hamedan, Iran.

\section{Authors' contributions}

AS performed the laboratory experiments and wrote the manuscript. AM designed the research, and edited the manuscript. TS and PM provided scientific consultations, and edited the manuscript.

\section{Acknowledgments}

The authors would like to thank Bu-Ali Sina University.

\section{References}

1. Bjarnsholt T. The role of bacterial biofilms in chronic infections. Apmis 2013;121:1-58. 
2. Khan J, Tarar SM, Gul I, Nawaz U, Arshad M. Challenges of antibiotic resistance biofilms and potential combating strategies: a review. 3 Biotech 2021;11:1-15.

3. Donlan RM. Biofilms: microbial life on surfaces. Emerging infectious diseases 2002;8:881.

4. Lewis K. Persister cells, dormancy and infectious disease. Nature Reviews Microbiology 2007;5:4856.

5. Yu D, Zhao L, Xue T, Sun B. Staphylococcus aureus autoinducer-2 quorum sensing decreases biofilm formation in an icaR-dependent manner. BMC microbiology 2012;12:1-12.

6. Sharifi A, Mohammadzadeh A, Zahraei Salehi T, Mahmoodi P, Nourian A. Cuminum Cyminum L. essential oil (CCEO): A promising antibacterial and anti-virulence agent against multidrug-resistant Staphylococcus aureus. Frontiers in Microbiology 2021;12:2016.

7. Sharifi A, Mohammadzadeh A, Zahraei Salehi T, Mahmoodi P. Antibacterial, antibiofilm and antiquorum sensing effects of Thymus daenensis and Satureja hortensis essential oils against Staphylococcus aureus isolates. Journal of applied microbiology 2018;124:379-88.

8. Francois P, Schrenzel J, Stoerman-Chopard C, Favre H, Herrmann M, Foster TJ, et al. Identification of plasma proteins adsorbed on hemodialysis tubing that promote Staphylococcus aureus adhesion. Journal of Laboratory and Clinical Medicine 2000;135:32-42.

9. Christensen GD, Simpson WA, Younger J, Baddour L, Barrett F, Melton D, et al. Adherence of coagulase-negative staphylococci to plastic tissue culture plates: a quantitative model for the adherence of staphylococci to medical devices. Journal of clinical microbiology 1985;22:996-1006.

10. Freeman D, Falkiner F, Keane $C$. New method for detecting slime production by coagulase negative staphylococci. Journal of clinical pathology 1989;42:872-4.

11. Seo Y-S, Lee DY, Rayamahji N, Kang ML, Yoo HS. Biofilm-forming associated genotypic and phenotypic characteristics of Staphylococcus spp. isolated from animals and air. Research in Veterinary Science 2008;85:433-8.

12. Yarwood JM, Bartels DJ, Volper EM, Greenberg EP. Quorum sensing in Staphylococcus aureus biofilms. Journal of bacteriology 2004;186:1838-50.

13. Van Houdt R, Michiels C. Biofilm formation and the food industry, a focus on the bacterial outer surface. Journal of applied microbiology 2010;109:1117-31.

14. Di Bonaventura G, Stepanović S, Picciani C, Pompilio A, Piccolomini R. Effect of environmental factors on biofilm formation by clinical Stenotrophomonas maltophilia isolates. Folia microbiologica 2007;52:86-90.

15. Regassa LB, Novick RP, Betley MJ. Glucose and nonmaintained pH decrease expression of the accessory gene regulator (agr) in Staphylococcus aureus. Infection and immunity 1992;60:3381-8.

16. Rohde H, Knobloch JK-M, Horstkotte MA, Mack D. Correlation of biofilm expression types of Staphylococcus epidermidis with polysaccharide intercellular adhesin synthesis: evidence for involvement of icaADBC genotype-independent factors. Medical microbiology and immunology 2001;190:105-12. 
17. Stepanović S, Vuković D, Hola V, BONAVENTURA GD, Djukić S, Ćirković I, et al. Quantification of biofilm in microtiter plates: overview of testing conditions and practical recommendations for assessment of biofilm production by staphylococci. Apmis 2007;115:891-9.

18. Dhanawade NB, Kalorey DR, Srinivasan R, Barbuddhe SB, Kurkure NV. Detection of intercellular adhesion genes and biofilm production in Staphylococcus aureus isolated from bovine subclinical mastitis. Veterinary research communications 2010;34:81-9.

19. Mathur T, Singhal S, Khan S, Upadhyay D, Fatma T, Rattan A. Detection of biofilm formation among the clinical isolates of staphylococci: an evaluation of three different screening methods. Indian journal of medical microbiology 2006;24:25-9.

20. Kennedy CA, O'Gara JP. Contribution of culture media and chemical properties of polystyrene tissue culture plates to biofilm development by Staphylococcus aureus. Journal of medical microbiology 2004;53:1171-3.

21. Knobloch JK-M, Horstkotte MA, Rohde H, Mack D. Evaluation of different detection methods of biofilm formation in Staphylococcus aureus. Medical microbiology and immunology 2002;191:1016.

22. Gerke C, Kraft A, Süßmuth R, Schweitzer O, Götz F. Characterization of theNAcetylglucosaminyltransferase Activity Involved in the Biosynthesis of the Staphylococcus epidermidisPolysaccharide Intercellular Adhesin. Journal of Biological Chemistry 1998;273:1858693.

23. Croes S, Deurenberg RH, Boumans M-LL, Beisser PS, Neef C, Stobberingh EE. Staphylococcus aureus biofilm formation at the physiologic glucose concentration depends on the $S$. aureus lineage. BMC microbiology 2009;9:1-9.

24. Vuong C, Saenz HL, Götz F, Otto M. Impact of the agr quorum-sensing system on adherence to polystyrene in Staphylococcus aureus. The Journal of infectious diseases 2000;182:1688-93.

25. Lim Y, Jana M, Luong TT, Lee CY. Control of glucose-and NaCl-induced biofilm formation by rbf in Staphylococcus aureus. Journal of bacteriology 2004;186:722-9.

26. Kobayashi N, Wu H, Kojima K, Taniguchi K, Urasawa S, Uehara N, et al. Detection of mecA, femA, and femB genes in clinical strains of staphylococci using polymerase chain reaction. Epidemiology \& Infection 1994;113:259-66.

27. He J-z, Wang A-q, Liu G, Gao J, Ali T, Han B. Biofilm Formation and Biofilm-Associated Genes Assay of Staphylococcus aureus Isolated from Bovine Subclinical Mastitis in China. Pakistan Veterinary Journal 2014;34.

28. Christensen GD, Simpson WA, Bisno AL, Beachey EH. Adherence of slime-producing strains of Staphylococcus epidermidis to smooth surfaces. Infection and immunity 1982;37:318-26.

29. Yazdani R, Oshaghi M, Havaei A, Pishva E, Salehi R, Sadeghizadeh M, et al. Detection of icaAD gene and biofilm formation in Staphylococcus aureus isolates from wound infections. 2006.

30. Livak KJ, Schmittgen TD. Analysis of relative gene expression data using real-time quantitative PCR and the $2-\Delta \Delta C T$ method. methods 2001;25:402-8. 
31. Harapanahalli AK, Chen Y, Li J, Busscher HJ, van der Mei HC. Influence of adhesion force on icaA and cidA gene expression and production of matrix components in Staphylococcus aureus biofilms. Applied and environmental microbiology 2015;81:3369-78.

32. Koprivnjak T, Mlakar V, Swanson L, Fournier B, Peschel A, Weiss JP. Cation-induced transcriptional regulation of the dlt operon of Staphylococcus aureus. Journal of Bacteriology 2006;188:3622-30.

\section{Figures}

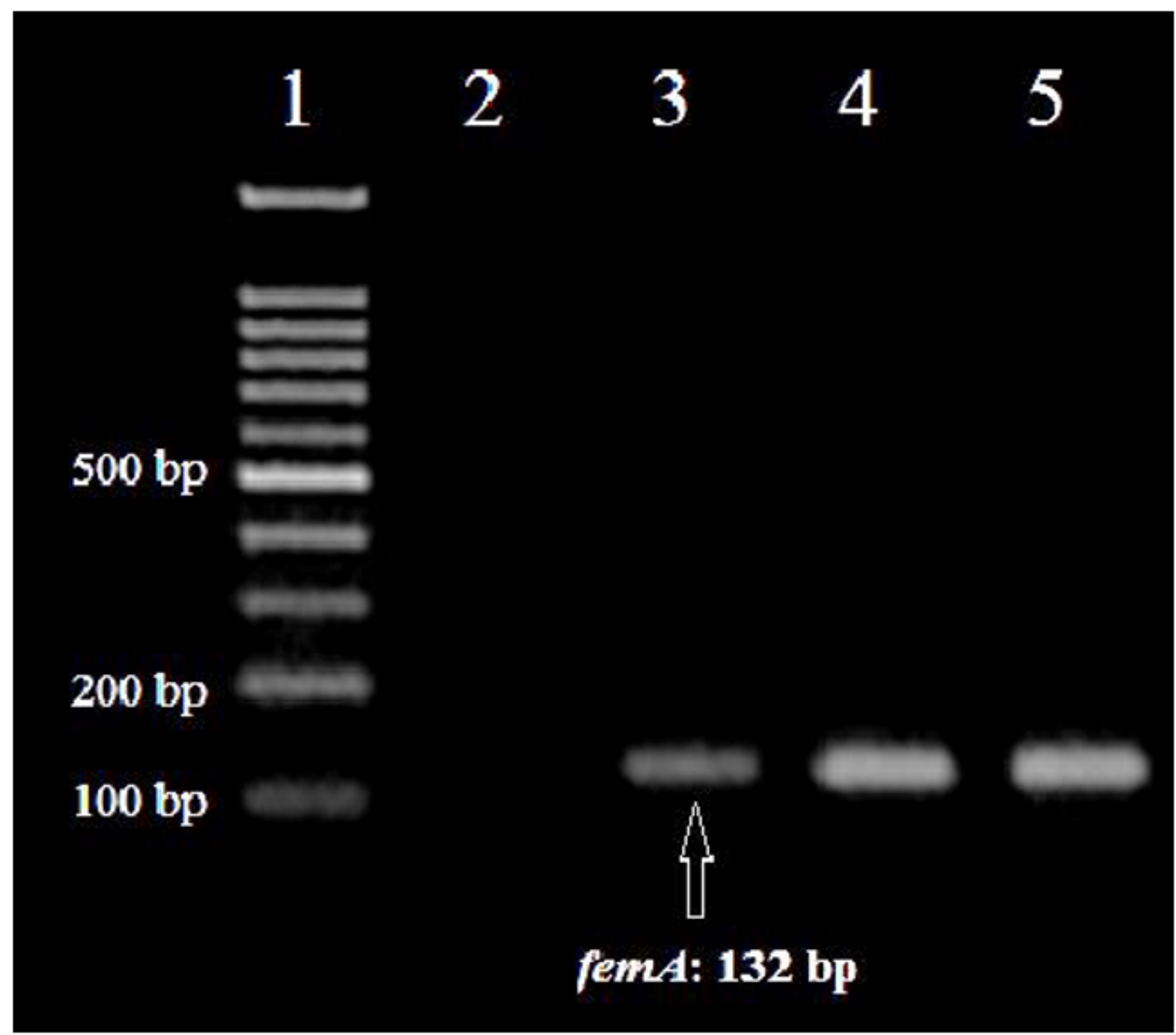

\section{Figure 1}

Agarose gel electrophoresis of species-specific PCR. Lane 1: 100 bp DNA ladder, lane 2: negative control (no template DNA), lane 3: positive control for femA gene (S. aureus ATCC 25923), lanes 4 and 5: PCR amplicons obtained from the isolates. 


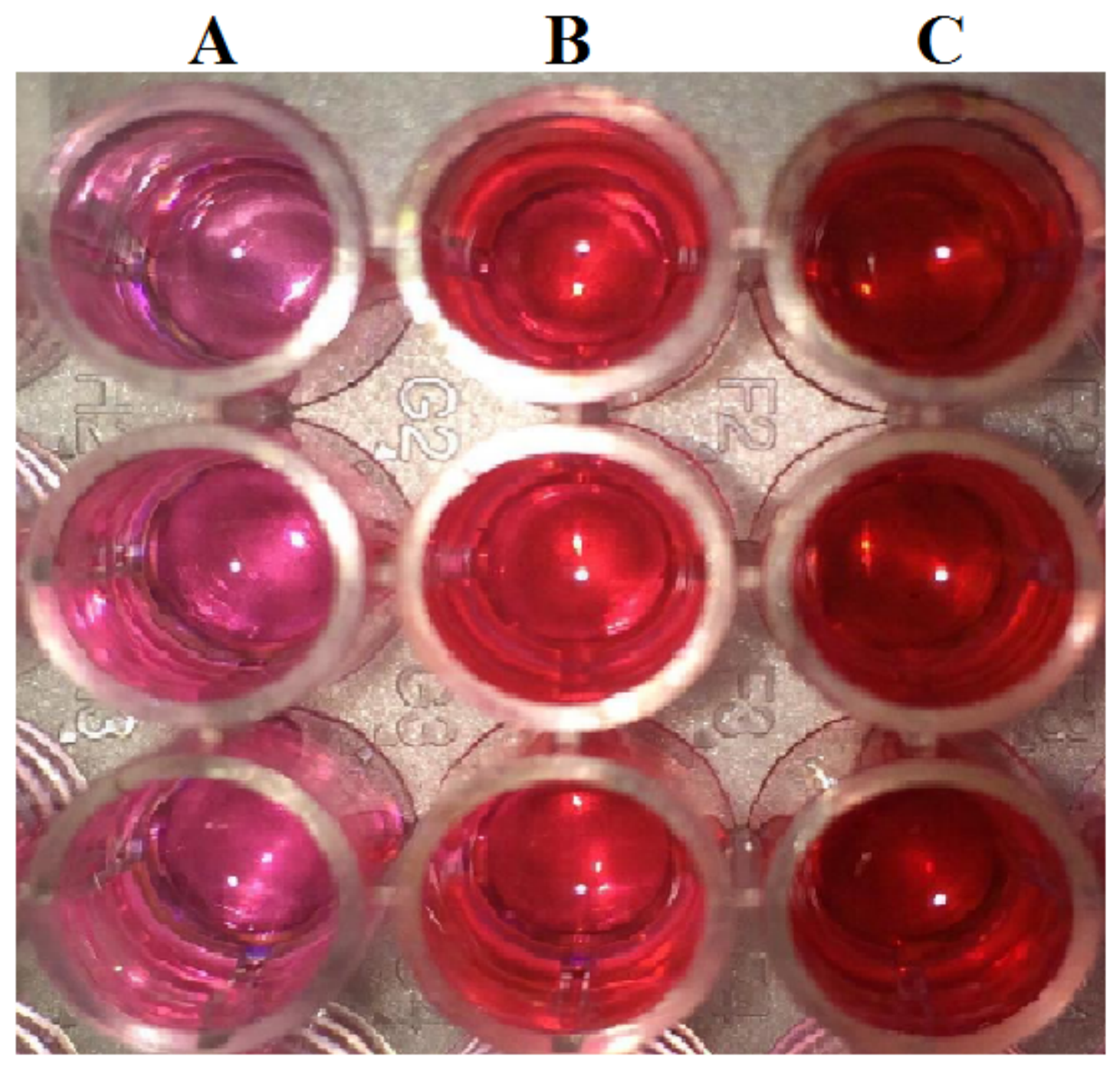

Figure 2

Measurement of the ability of biofilm formation using microtiter plate assay (MtP). Lanes A to $\mathrm{C}$ indicate the reaction of weak, moderate and strong biofilm producers, respectively. 


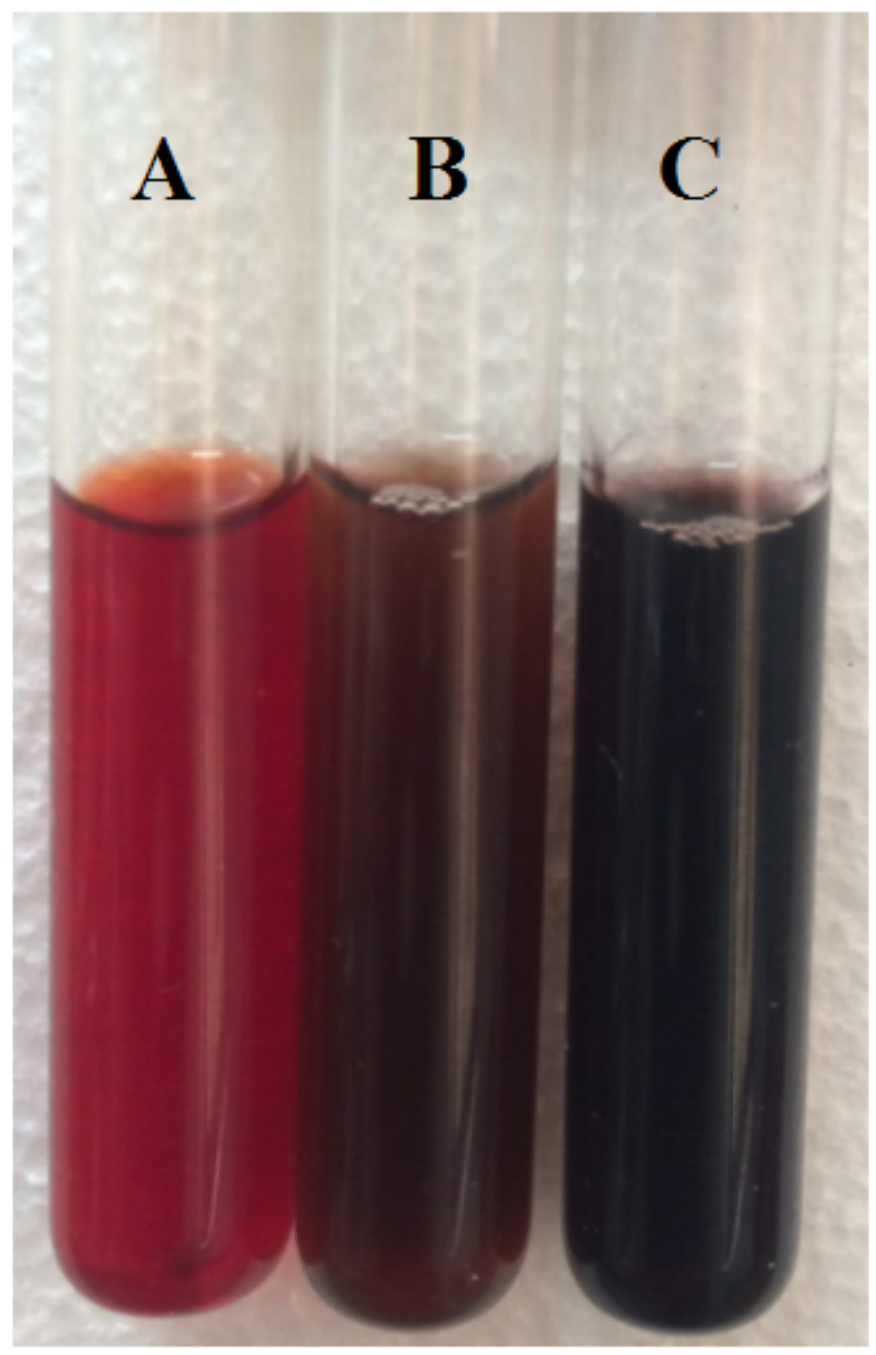

\section{Figure 3}

Screening of slime (PIA) producers by tube test. A: negative slime producer (S. epidermidis ATCC 12228, red pigments), B: moderate slime producer, and C: positive slime producer (S. aureus ATCC 25923, black pigments). 


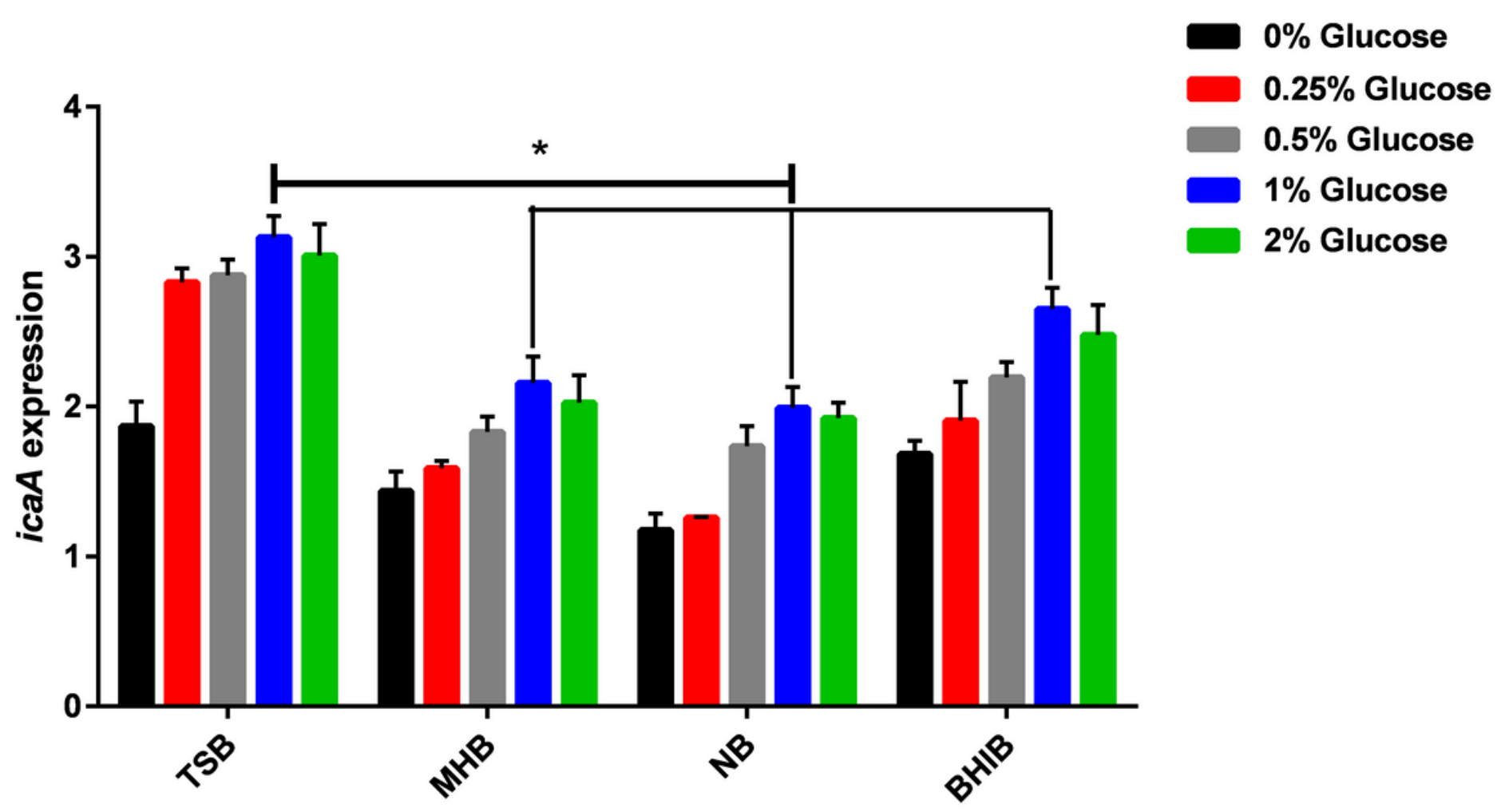

Tested media with different glucose concentrations

Figure 4

The icaA expression in various media contained $0,0.25,0.5,1$ and $2 \%$ glucose. The maximum icaA expression has occurred in TSB supplemented with $1 \%$ glucose. The error bars represent the standard deviation of three replicates $(*$ : $P<0.05)$. 